\title{
Large Subcapsular Hematoma of the Spleen Complicating Acute Pancreatitis
}

Splenic complications are rare in cases of acute or chronic pancreatitis. Splenic complications associated with acute and chronic pancreatitis include splenic vein thrombosis, arterial pseudoaneurysms, splenic hematoma and splenic rupture. Splenic hematoma is a rare complication of acute or chronic pancreatitis when compared with traumatic origin of subcapsular hematoma. Local factors such as thrombosis of the splenic artery or veins, intrasplenic pseudocysts, perisplenic adhesions, enzymatic digestion and coagulation disorders may play a role in the pathogenesis of splenic hematoma.

We report a case of a 17 years old boy who presented to Emergency Department with complain of severe pain abdomen, vomiting and weakness for two days. He complained of pain abdomen off and on for last one year and vomiting and weakness for last two month. He gave history of taking alcohol three to four times a week for last two years. On examination he was haemodynamically stable but was severely pale. On blood investigation serum amylase and lipase were significantly raised. His $\mathrm{Hb}$ was $5 \mathrm{gm} \%$. CECT of abdomen revealed features of Pancreatitis and large subcapsular perisplenic haematoma [Table/Fig-1]. It also shows splenic vein thrombosis [Table/Fig-2]. He was managed with intravenous fluid, blood transfusions, prophylactic antibiotic and pain killers. Patient responded well to the conservative management. His blood parameters improved and perisplenic haematoma showed gradual resolution on serial USG examination [Table/Fig-3]. Patient discharged in stable condition and is being followed up clinically and by serial USG examination. He is doing well after six months of follow-up.

Splenic complications are rare in cases of acute or chronic pancreatitis [1]. Anatomically pancreatic tail is close to spleen. This position leads to involvement of spleen in cases of pancreatitis. Splenic complications associated with pancreatitis are splenic vein thrombosis, arterial pseudo aneurysms, splenic hematoma and splenic rupture. Such complications occur in $1-5 \%$ of pancreatitis [2]. The mechanisms leading to these complication are: (1) splenic vessel thrombosis and pseudo aneurysm formation; (2) dissection of a pancreatic pseudo cyst into the hilum of the spleen; or (3) extension of the inflammatory process from the tail of the pancreas into the hilum of the spleen. Splenic hematoma is a rare complication of acute or chronic pancreatitis. Thrombosis of the splenic artery or veins, intrasplenic pseudo cysts, perisplenic adhesions, enzymatic
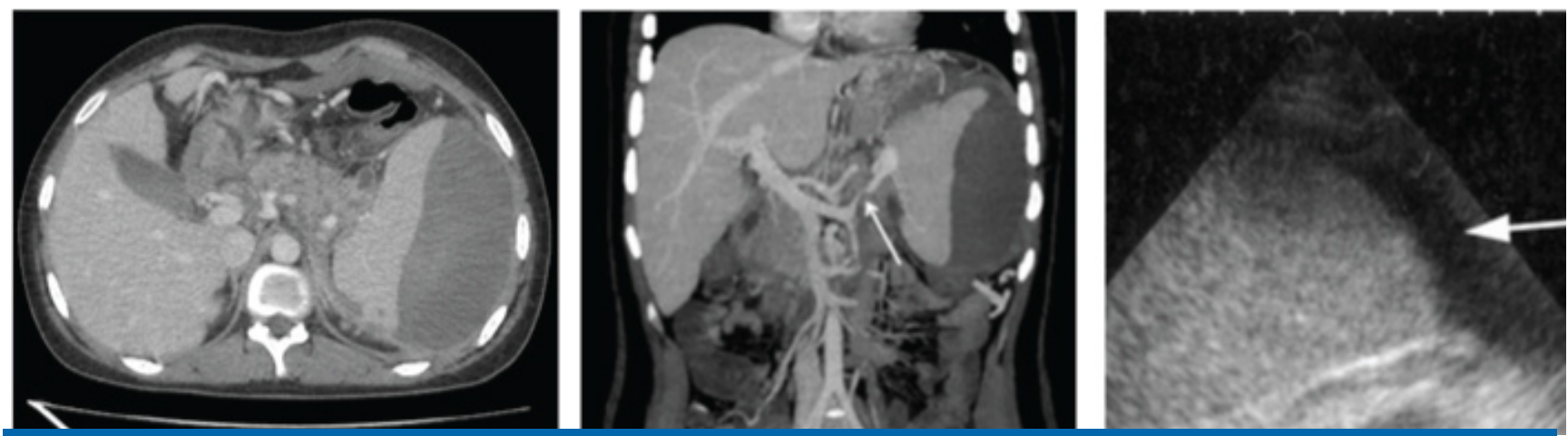

[Table/Fig-1]: CECT showing subcapsular splenic haematoma. [Table/Fig-2]: CECT showing splenic vessel thrombosis. [Table/Fig-3]: USG showing resolving splenic subcapsular haematoma. 
digestion and coagulation disorders play a role in the pathogenesis of splenic hematoma. The splenic hematoma in our case was probably caused by the splenic vein thrombosis as demonstrated on abdominal CT Angiography. The hematoma that develops in this situation is contained within the splenic capsule. Because splenic involvement in patients with acute or chronic pancreatitis is uncommon, the diagnosis of subcapsular hematoma of the spleen needs the alertness of physicians and imaging studies. Patients with pancreatitis exhibiting a mass in the left upper quadrant, pain radiating to the left shoulder, elevation of the left diaphragm, and a moderate fall in hematocrit should be suspected to have splenic complications. Abdominal CT should be performed early in questionable patients. A splenic hematoma can be distinguished from simple fluid collection based on density (Hounsfield units > 30) [3]. The management of subcapsular splenic hematoma in pancreatitis is not established. Surgical treatment option includes splenectomy and percutaneous drainage. In cases of large splenic hematoma (> $5 \mathrm{~cm}$ ) as a complication of pancreatitis, pressure reduction by percutaneous drainage or laparotomy should be administered as early as possible. Some reports advocates aggressive management with early splenectomy to avoid splenic rupture. Percutaneous drainage for splenic subcapsular collections may be a feasible treatment, but there are only a few reports of it in the literature [4]. Some authors suggested that most splenic complications of pancreatitis regress spontaneously and may be managed conservatively. Surgical indication is based on clinical findings. They suggested that conservative approach to the management of splenic subcapsular hematoma is appropriate when the Patient is haemodynamically stable and is showing improving symptoms and signs [5].

\section{REFERENCES}

[1] Malka D, Hammel P, Levy P, Sauvanet A, Ruszniewski P, Belghiti $J$, Bernades P. Splenic complications in chronic pancreatitis: prevalence and risk factors in a medical-surgical series of 500 patients. Br J Surg. 1998;85:1645-49.

[2] Thompson JE Jr, Ashley SW. Subcapsular hematoma of the spleen associated with acute pancreatitis. Surgery. 1997;121: 231-33.

[3] Rypens F, Deviere J, Zalcman M, Braude P, et al. Splenic parenchymal complications of pancreatitis: CT findings and natural history. J Comput Assist Tomogr.1997;21:89-93.

[4] Siu TL. Percutaneous drainage of spontaneous subcapsular haematoma of the spleen complicating chronic pancreatitis. Surgeon. 2004;2:52-55.

[5] Vyborny CJ, Merrill TN, Reda J, Geurkink RE, Smith SJ. Subacute subcapsular hematoma of the spleen complicating pancreatitis: successful percutaneous drainage. Radiology. 1988; 169:161-62.
AUTHOR(S):
1. Dr. Manish Kumar Pandey
2. Dr. Utpal Anand
3. Dr. Ramesh Kumar

\section{PARTICULARS OF CONTRIBUTORS:}

1. Consultant, Department of Surgery, Paras HMRI Hospital, Patna, India.

2. Consultant Gastro Surgery, Department of Surgery, Paras HMRI Hospital, Patna, India.

3. Consultant, Department of Gastroenterology, Paras HMRI Hospital, Patna, India.
NAME, ADDRESS, E-MAIL ID OF THE CORRESPONDING AUTHOR:

Dr. Manish Kumar Pandey, 102 B, Luv Kush Apartment, Lohiya Path, Jagdeo Path, Patna-800014, India.

E-mail: drmanish99@gmail.com

FINANCIAL OR OTHER COMPETING INTERESTS: None. 